\title{
PMTCT Cascade in Delhi -Are We Ready to Launch New WHO PMTCT Strategy to Eliminate Pediatric HIV?
}

\author{
${ }^{1}$ Dr.A.K. Gupta, ${ }^{2}$ Dr. D. Somashekar, ${ }^{3}$ Mr. Girraj Pratap, ${ }^{4}$ Mr. B.C. Joshi, \\ ${ }^{5}$ Mr.P.B.Kaushik, ${ }^{6}$ Mr. S. Chakraborty, ${ }^{7}$ Mr. Kuldeep Rai \\ ${ }_{1,2,3,4,5,6,7}$ Delhi State AIDS Control Society, Government of Delhi, Dr. B.S.A. Hospital Complex, Sector VI, \\ Rohini, Delhi-110085, India
}

\begin{abstract}
Government of India has planned to adopt option ' $B$ ' of the WHO PMTCT guidelines to be initially piloted in few high HIV prevalent states of the country in September 2012. The present study was undertaken with aim to assess level of preparedness of Delhi to launch the new WHO (2010) PMTCT strategy. Gaps were identified at each step of the PMTCT cascade during January 2011 to December 2011 which could be successfully addressed by simple measures like sensitizing pediatricians and obstetricians \& gynecologists, review meeting with PPTCT /ICTC staff, expanding the outreach activity, training ANMs, on site linkage meetings and scaling up Early Infant Diagnosis programme. The stage is now set for the launch of new WHO PMTCT guidelines of 'Triple ARV prophylaxis to all HIV positive pregnant women with CD4 count > 350 cells / cu.mm. to achieve the global objective of virtual elimination of pediatric HIV by 2015. However, the new strategy would require comprehensive convergence with $M C H$ and remarkable commitment from the pediatricians, obstetricians \& gynecologists, counselors and ANMs involved in the care of pregnant women to ensure adherence to the triple ARV regimen to prevent emergence of drug resistance.
\end{abstract}

Keywords - Prevention of Mother to Child Transmission, PMTCT cascade, Eliminating Pediatric HIV

\section{Introduction:}

Prevention of Mother to Child Transmission (PMTCT) is a dynamic and rapidly developing field. Without any PMTCT intervention the reported MTCT rate average 35\% [1]. WHO have issued PMTCT guidelines from time to time and left for the countries to adopt them based on their resources and assessing the feasibility. WHO PMTCT 2010 ARV guidelines on treating pregnant women and preventing infections in infants were a major step towards more efficacious regimens wherein WHO emphasized the importance of lifelong ART to all HIV infected pregnant women with CD4 count $\leq 350$ cells/cu.mm and recommended two short term ARV prophylaxis regimens (option A and Option B) for women with CD4 count above 350 [2]. UNICEF has now set the stage for elimination of pediatric HIV infections by issuing guidance to adopt a single, universal regimen both to treat HIV infected pregnant women and to prevent MTCT of HIV, termed Option B+ [3]. National task Force under aegis of NACO, MoHFW, Government of India has planned to adopt option B of the WHO guideline to be initially piloted in some high HIV prevalent states of the country in September 2012. The present study was conducted with the aim to assess the level of preparedness of Delhi to launch the new WHO (2012) PMTCT ARV guidance of option B.

\section{Methodology}

The cascade of PMTCT services delivered through 19 PPTCT and 29 ICT centers in Delhi include offering HIV counseling and testing to all pregnant women (PW), counseling and testing of spouses of PW detected HIV infected, registration of HIV infected PW in HIV care \& CD4 count at ART centre, initiation of ART if CD4 count is $\leq 350$ cells/cu.mm and PW with CD4 above 350 are offered Single dose NVP prophylaxis to mom during labour and to the infant within 72 hours of birth. Besides these, PW is also educated for positive prevention by promoting safe sexual practices using condoms, preventing unwanted pregnancies by linking to family planning services under RCH programme and diagnosis and treatment of sexually transmitted infections (STI) at STI clinics run under the national AIDS Control Programme. Safe infant feeding counseling is done during pregnancy, after birth, at six weeks and during weaning at around six months of age The HIV exposed infant born enter the Early Infant Diagnosis (EID) cascade at minimum 6 weeks of age for DNA PCR test by Dried Blood Spot (DBS) \& initiation of Cotrimoxazole Prophylaxis Therapy (CPT) to prevent opportunistic infections. Tracking of clients lost to follow up at any stage of the cascade is done by PPTCTC counselors. In addition, 14 Outreach workers (ORWs) have been deployed at 14 PPTCT centers through GFATM funded project to follow-up HIV positive PW and HIV exposed infants.

Data pertaining to PMTCT programme was gathered from all counseling and testing centers during the period from $1^{\text {st }}$ January 2011 to $31^{\text {st }}$ December 2011 to evaluate the performance at each step of PMTCT 
cascade. Further, following activities were undertaken in Delhi during February 2012 to March 2012 to strengthen PPTCT programme in Delhi:

- Review meeting with PPTCT /ICTC staff to strengthen PPTCT and EID cascade.

- Sensitization of Pediatricians and O\&G specialist of 48 Govt Hospitals of Delhi on the new WHO PMTCT (Option B) regimen.

- Training of 600 ANMs of NRHM to scale up PPTCT programme with aim of involving ANMs in follow up of HIV positive PW \& HIV exposed infants,

- On site sensitization programme to improve linkages of 48 PPTCT centers/ICTC with 9 ART centers

- Expanding outreach activity of GFATM funded ORWs-Earlier 14 ORWs were deployed at 14 PPTCTCs to track HIV positive pregnant \& HIV exposed infant who were lost to follow up. April 2012 onwards, their outreach activity was extended to 48 PPTCTC/ICTCs by increasing the number of ORWs to 19 and allotting 2-3 centers per ORW.

- Refresher trainings of PPTCT and ICTC counselors with emphasis of filing gaps in the PMTCT cascade.

- Scaling up Early Infant Diagnosis (EID) by DBS DNA PCR test by increasing the number of EID sites from 19 to 27.

\section{Results}

PMTCT cascade during January 2011 to December 2011 is shown in Fig 1. Against the annual target of 2 lakh pregnant women to be counseled and tested in the study period, 187779 (93.9\%) pregnant women were counseled and tested. Of this, $10384(5.5 \%)$ pregnant women did not attend post test counseling and failed to receive test results. Of the $331(0.18 \%)$ pregnant women detected HIV positive, $325(98.2 \%)$ attended post test counseling, $264(81.23 \%)$ got registered at ART centre, and 194 (73.5\%) underwent CD4 count. Of the 84 pregnant women with CD4 count $\leq 350$ cells/ cum mm, $51(60.7 \%)$ were put on ART during pregnancy. $29 \mathrm{HIV}$ positive pregnant women underwent MTP, 47 migrated to their native states for delivery, 7 delivered at home and 248 delivered in an Institution in Delhi. 161 (64.9\%) Mother Baby (MB) pairs received ARV prophylaxis for PMTCT and safe infant feeding counseling after birth. 166 (66.94\%) HIV exposed infants were enrolled for EID and initiated Cotrimoxazole Prophylaxis Therapy (CPT).

Further, 33 (39.3\%) HIV positive pregnant women eligible for ART could not be initiated on anti retroviral treatment. 248(74.92\%) HIV positive pregnant women delivered in an institution of which 202(81.45\%) delivered in Govt. hospitals. However, 41 (20.3\%) MB pair taken care of in Govt hospitals did not receive ARV prophylaxis. Also, $36(17.8 \%)$ HIV exposed infants born in Govt hospitals failed to enter EID cascade.

The PMTCT data was examined during April 2012 to June 2012 after taking corrective measures in Feb - March 2012. The results are presented in Fig 2. Against target of 50000 pregnant women to be counseled and tested during this period, $47216(94.4 \%)$ were counseled and tested. Of the $83(0.18 \%)$ pregnant women detected HIV positive, all attended post test counseling and received HIV test results, $70(84.30 \%)$ got registered at antiretroviral treatment centre and $67(95.7 \%)$ underwent CD4 tests. $25(78.12 \%)$ of the 32 with CD4 $\leq 350$ cells per cu mm were put on ART. 48 (57.83\%) HIV positive pregnant women delivered in Institution and all MB pairs received ARV prophylaxis under PMTCT, 5 HIV positive pregnant women underwent MTP while expected date of delivery of $40 \mathrm{HIV}$ pregnant women is still due. All $48 \mathrm{HIV}$ exposed infants have been enrolled for EID and initiated CPT within 2 months after birth.

\section{Discussion:}

Gaps identified in the PMTCT cascade for the year 2011cascade could be successfully addressed by simple measures like sensitizing pediatricians and obstetricians \& gynecologists of 45 Govt Hospitals of Delhi, review meeting with PPTCT /ICTC staff to fill up the gaps, expanding the outreach activity to cover all PPTCT and ICT centers through ORWs deployed under GFATM project, training of 600 ANMs of NRHM and on site meetings to strengthen linkages of PPTCT/ICT centers with ART centers. 9 New EID sites were established thereby helping to achieve 100\% enrollment of HIV exposed infants for early Infant Diagnosis by DBS DNA PCR and initiation of CPT to prevent opportunistic infections. This corroborates with previous recommendation of convergence of PMTCT programme with MCH programme as a comprehensive PMTCT strategy [4]. Mother Child Tracking System of National Rural Health Mission is also being explored in Delhi to ensure "continuum to care' of HIV positive PW or HIV exposed infants by utilizing services of 600 trained ANMs.

The stage is now set for adopting the WHO revised PMTCT guidelines (2010) in Delhi which will be helpful for mother's own health as well as in reducing the mother to child transmission to less than $5 \%$ in line with the global objective of virtual elimination of pediatric HIV by 2015 [1,3]. However, adapting the new guidelines will require ensuing $100 \%$ adherence to triple ARV PMTCT regimen to prevent emergence of drug resistance. Initiating triple ARV therapy in pregnancy may of its own help in maintaining close contact of pregnant women with the PPTCT team since ARV drugs will be issued on monthly basis. During each visit 
pregnant women can be counseled for drug adherence, ensuring institutional delivery, ARV prophylaxis to the infant and follow up of MB pair up to 18 months of age.

\section{Conclusion}

PMTCT programme can be strengthened by addressing gaps in the PMTCT cascade through convergence with $\mathrm{MCH}$ programme and sensitizing and training staff involved in maternal and child health care. This convergence will be helpful in adopting new WHO Triple ARV prophylaxis PMTCT guidelines.

\section{References}

[1] Towards the elimination of mother to child transmission of HIV. Report of a WHO technical consultation, November 2010, Geneva, Switzerland. Available at: http://www.who.int/hiv/pub/mtct/elimination_report/en/index.html (12 March 2012, date last accessed).

[2] WHO. Antiretroviral drugs for treating pregnant women and preventing HIV infection in infants: Recommendations for a public health approach 2010 version. Available at : http://whqlibdoc.who.int/publications/2010/9789241599818_eng.pdf (10 July 2011, date last accessed).

[3] UNICEF. A business case for options B and B+ to eliminate mother to child transmission of HIV by 2015 Revised July 2012. Available at: http://www.unicef.org/aids/files/DISCUSSION_PAPER.A_BUSINESS_CASE_FOR_OPTIONS_B.pdf (12 March 2012, date last accessed).

[4] Prevention of Mother-to-Child Transmission of HIV: Expert Panel Report and Recommendations to the U.S. Congress and U.S. Global AIDS Coordinator January 2010. Available at : http://www.pepfar.gov/documents/organization/135465.pdf (14th March 2012, date last accessed )

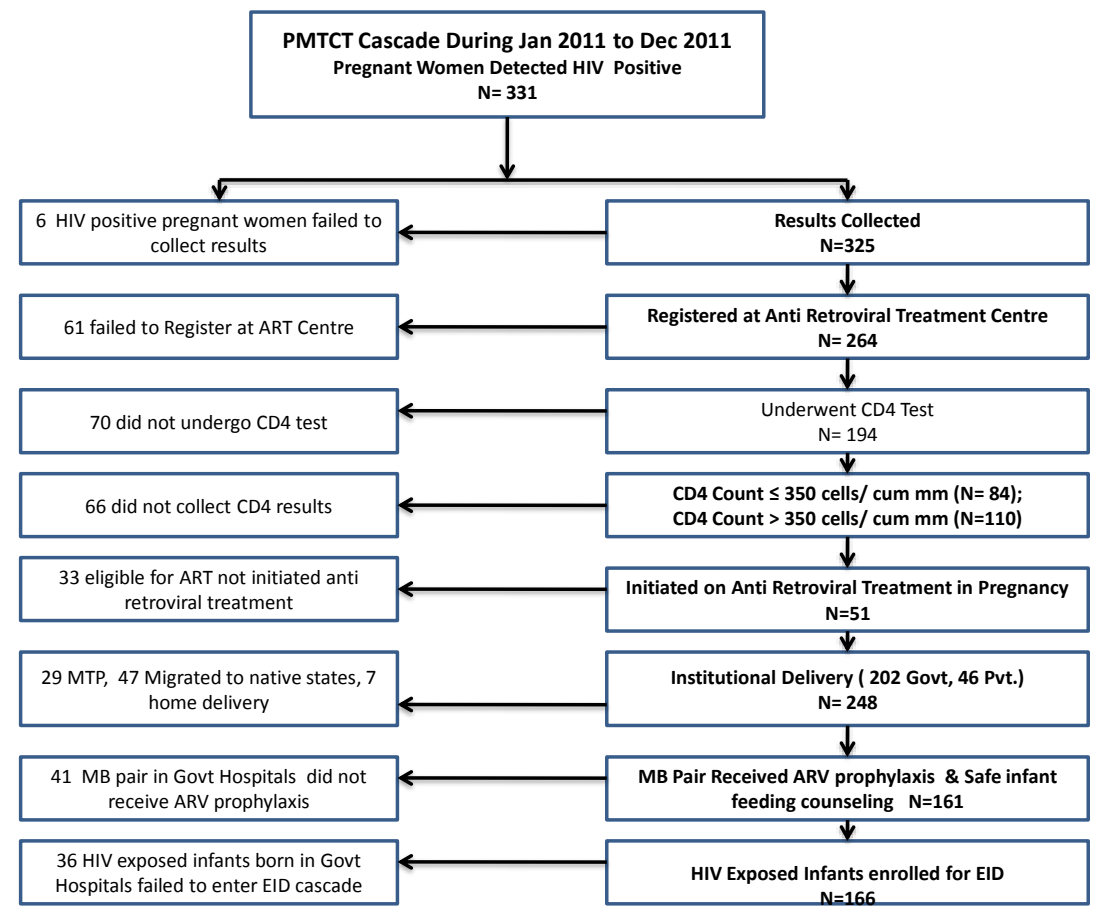

Fig 1: PMTCT cascade from January 2011 to December 2011

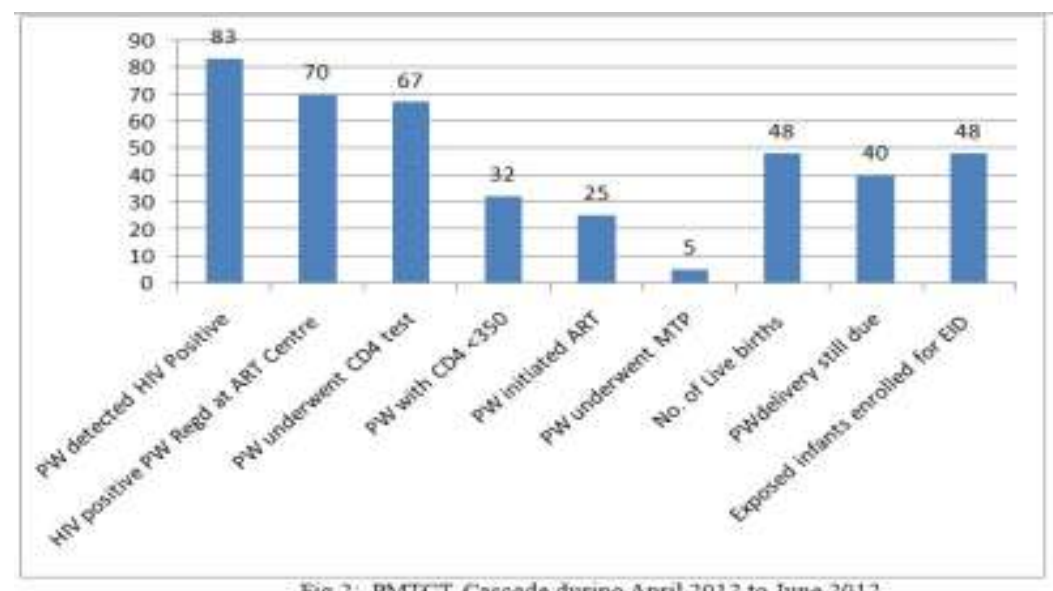

Fig 2: PMTCT Cascade during April 2012 to June 2012 